\title{
Payer Mix and EHR Adoption in Hospitals
}

By: Shin, Dong Yeong, Menachemi, Nir, Diana, Mark, Kazley, Abby Swanson, PhD, Ford, Eric $\mathrm{W}, \mathrm{PhD}$

Shin, D. Y., Menachemi, N., Diana, M., Kazley, A. S., \& Ford, E. W. (January 01, 2012). Payer mix and EHR adoption in hospitals. (electronic health records)(Report). Journal of Healthcare Management, 57(6), 449-50.

Made available courtesy of Health Administration Press for the American College of Healthcare Executives: http://www.ache.org/pubs/jhmsub.cfm

***Reprinted with permission. No further reproduction is authorized without written permission from Health Administration Press. This version of the document is not the version of record. It has not been edited or typeset and it does not contain the Practitioner Application that accompanies the article in the published issue. Exhibits 1-4 have been omitted from this formatted document****

\section{Abstract:}

Payers are known to influence the adoption of health information technology (HIT) among hospitals. However, previous studies examining the relationship between payer mix and HIT have not focused specifically on electronic health record systems (EHRs). Using data from the Nationwide Inpatient Sample and the American Hospital Association Annual Survey, we examine how Medicare, Medicaid, commercial insurance, and managed care caseloads are associated with EHR adoption in hospitals. Overall, we found a weak relationship between payer mix and EHR adoption. Medicare and, separately, Medicaid volumes were not associated with EHR adoption. Furthermore, commercial insurance volume was not associated with EHR adoption; however, a hospital located in the third quartile of managed care caseloads had a decreased likelihood of EHR adoption. We did not find empirical evidence to support the hypothesis that payer generosity and other indirect mechanisms influence EHR adoption in hospitals. The direct incentives embedded in the Health Information Technology for Economic and Clinical Health Act may have a positive influence on EHR adoption - especially for hospitals with high Medicare and/or Medicaid caseloads. However, it is still uncertain whether the available incentives will offset the barriers many hospitals face in achieving meaningful use of EHRs.

\section{Keywords:}

Hospital administration | healthcare management | electronic health record systems | health information technology

Article: 


\section{EXECUTIVE SUMMARY}

Payers are known to influence the adoption of health information technology (HIT) among hospitals. However, previous studies examining the relationship between payer mix and HIT have not focused specifically on electronic health record systems (EHRs). Using data from the Nationwide Inpatient Sample and the American Hospital Association Annual Survey, we examine how Medicare, Medicaid, commercial insurance, and managed care caseloads are associated with EHR adoption in hospitals. Overall, we found a weak relationship between payer mix and EHR adoption. Medicare and, separately, Medicaid volumes were not associated with EHR adoption. Furthermore, commercial insurance volume was not associated with EHR adoption; however, a hospital located in the third quartile of managed care caseloads had a decreased likelihood of EHR adoption. We did not find empirical evidence to support the hypothesis that payer generosity and other indirect mechanisms influence EHR adoption in hospitals. The direct incentives embedded in the Health Information Technology for Economic and Clinical Health Act may have a positive influence on EHR adoption - especially for hospitals with high Medicare and/or Medicaid caseloads. However, it is still uncertain whether the available incentives will offset the barriers many hospitals face in achieving meaningful use of EHRs.

\section{INTRODUCTION}

Research has shown that payer mix, defined as the combination of thirdparty payers that makes up a hospital's book of business, can influence hospitals' strategic behaviors. Studies have found that higher percentages of Medicaid (Cleverley and Harvey 1992; McKay and Deily 2005) or Medicare patients (Rosko 2001) are negatively associated with financial performance. Furthermore, given that varying reimbursement rates are negotiated in the private insurance book of business, hospital revenue per admission has been demonstrated to predict operational efficiency (Dor and Farley 1996; McKay and Deily 2005) and clinical performance (Clement and Grazier 2001; Menachemi et al. 2007). Attempting to leverage the influence that the public insurance programs have on hospitals, the federal government, through the Health Information Technology for Economic and Clinical Health (HITECH) Act of 2009, aims to increase the adoption and "meaningful use" of electronic health record systems (EHRs) by providing incentives and penalties to hospitals through the Medicare and Medicaid programs (CMS 2010).

The HITECH Act, part of federal stimulus legislation, allocated billions of dollars in incentive payments to providers to facilitate the adoption and use of EHR technology (Blumenthal and Tavenner 2010). The incentive payments, made to hospitals that adopt, implement, upgrade, or successfully demonstrate their meaningful use of certified EHRs, are available as of fiscal year 
2011. Hospitals that do not achieve meaningful use by 2015 are potentially subject to Medicare and Medicaid payment penalties of up to 2 percent in later years (CMS 2010).

The relationship between hospitals' EHR adoption rates and payer mix is not fully understood. However, several researchers have found that payer mix is correlated with health information technology (HIT) adoption. Based on 2004 data, Furukawa and colleagues (2008) found that the adoption of computerized physician order entry systems, but not EHR systems, was associated with an increased rate of Medicare patients as a percentage of all discharges. Similarly, McCullough (2008) found that the adoption of pharmacy information systems was positively related to Medicare caseload. The McCullough study also found that the adoption of laboratory information systems and radiology information systems was negatively associated with higher levels of Medicaid caseloads. Lastly, evidence from Florida hospitals suggests that an increase in privately insured patients is positively associated with the number of HIT applications adopted (Menachemi et al. 2007). While the Florida study did not examine EHR adoption per se, differences in public insurance (e.g., Medicare, Medicaid) as a percentage of a hospital's discharges were not correlated with overall HIT adoption in that study. Therefore, the relationship between Medicare and Medicaid programs and EHR adoption rates is unclear.

The purpose of this article is to examine the relationship between acute care hospitals' payer mix and their EHR adoption rates. The study uses national data and a series of statistical analyses including chi-square, logistic regression, and multinomial regression to explore these relationships. A better understanding of how payer mix and EHR adoption are related will allow hospital leaders and public policymakers to capitalize on the legislative intent of the HITECH Act, which utilizes the relationship between payer mix (e.g., Medicare and/or Medicaid payer mix) and HIT adoption to directly motivate hospitals to use EHR technology. Our findings will also benefit those trying to better understand the impact that the meaningful use program may have on hospitals' EHR use. Moreover, our study will help hospital decision makers to better gauge their overall progress on EHR adoption relative to a national cohort of hospitals with similar payer mix combinations.

\section{CONCEPTUAL FRAMEWORK}

Payer mix may influence EHR adoption in two ways. The first is related to the concept of payer generosity; the second is related to certain reimbursement mechanisms inherent to some payers. Payer generosity refers to the relative payments a given insurance company provides for a given procedure or diagnosis. In general, certain payers are believed to reimburse less generously than others (Friedman et al. 2004). Specifically, government payers, such as Medicare and Medicaid, 
tend to provide lower reimbursement rates than do private payers, such as traditional indemnity insurance plans (Dobson, Davanzo, and Sen 2006). Given the negative relationship between the proportion of Medicare and Medicaid patients and hospital operating margin (e.g., Rosko 2001), the lower reimbursement rates of public insurance programs may not cover the entire cost of patient care (AHA 2010). This shortfall could lead to decreased operating margin in hospitals, thus leaving diem with less financial flexibility to consider major capital investments.

Researchers have noted that upfront capital requirements and high maintenance costs are the primary barriers to EHR adoption in acute care US hospitals (Jha et al. 2009). All other factors being equal, hospitals with higher proportions of Medicare and Medicaid patients are less likely to have the financial wherewithal to make die large capital investments necessary to buy an EHR system compared to facilities with higher percentages of private-pay patients. Thus, we would expect that a high amount of publicly insured patients is associated with a decreased likelihood of EHR adoption in hospitals.

Second, reimbursement mechanisms may play a role in EHR adoption. Under capitation arrangements and other prospective payment contracts, hospitals are financially motivated to improve efficiencies and lower costs in order to maximize profits. Under capitated reimbursement conditions, common among health maintenance organizations (HMOs), hospitals are paid a set amount for each enrolled person assigned regardless of the number or type of services provided to the person in a given time period (Mello, Stearns, and Norton 2002; Miller and Luft 2002). Many proponents of EHRs have claimed that EHRs will increase organizational efficiency while reducing duplication of effort (Brader 2005). To the extent that EHR adoption is viewed as a strategy for improving efficiencies and lowering costs (Ali et al. 2005; Boger 2003; Garrido et al. 2004), it may be pursued at higher rates by hospitals with a relatively higher number of HMO patients.

\section{METHODS}

We used a cross-sectional design with secondary data and with the acute care hospital as the unit of analysis. The analysis combines data from HCUP's (Healthcare Cost and Utilization Project) Nationwide Inpatient Sample (NIS) for 2007, the 2008 American Hospital Association (AHA) Annual Survey database, and the 2007 Medicare Cost Reports. We drew hospital discharge data from the 2007 NIS, while the data indicating EHR adoption status and organizational characteristics of hospitals were drawn from the 2008 AHA database. In addition, because of the relatively small sample size we obtained from the NIS, we performed a parallel analysis using the limited payer mix variables, but with a much larger sample size, from the AHA Annual Survey. Doing so allows us to robustly examine the relationship between payer mix and EHR adoption. 
The AHA database contains organizational information on US hospitals and on EHR systems use, which the AHA added to its annual assessment beginning in 2007 (Jha et al. 2009). The dependent variable is a categorical measure representing (a) fully implemented EHR, (b) partially implemented EHR, and (c) no EHR implemented in response to the following AHA survey question: "Does you hospital have an electronic health record?" Data measuring payer mix were extracted from the NIS database, which is the largest all-payer inpatient database in the United States and contains all discharge data from approximately a 20 percent stratified sample of community hospitals. The NIS database is frequently employed by health services researchers interested in hospital management and related issues (Boxer et al. 2003; LaPar et al. 2010; Russell et al. 2006).

To operationalize payer mix, we calculated the percentage of discharges for each hospital that were covered by each of the following primary payers: (1) Medicare, (2) Medicaid, (3) commercial indemnity insurance, (4) managed care organizations, and (5) all other payers. Of the 646 hospitals in the 2007 NIS data set that include AHA identifiers, 392 hospitals (60.7 percent) included all the information necessary to construct our main independent variables. We compared the excluded hospitals to our sample for validity purposes.

Given the loss in sample size that occurred as a result of merging with the NIS data, we also examined the relationship between Medicare caseloads and Medicaid caseloads using the AHA Annual Survey data. The AHA sample is much larger than the NIS but is limited to information on only two payers' caseloads (i.e., Medicare and Medicaid). By examining the relationships of interest with the NIS (comprehensive payer data limited to a small sample) and the AHA survey (limited payer data on a comprehensive sample size), we believe we were able to robustly examine the relationship between EHR adoption and payer mix.

\section{Variables}

To compute each payer mix variable in the NIS, we conducted a series of aggregations and calculations. First, we aggregated from the patient level to the hospital level the total number of discharges paid in each category of primary payers in each hospital from the Inpatient Core Files. Next, we merged this aggregated data set with the Hospital Weights Files to compute the proportion of discharges paid for each payer type for each hospital (i.e., the total number of discharges paid by each payer divided by the total number of discharges in a hospital). Each payer mix variable in the data set was converted into quartiles to facilitate ease of interpretation. 
The result is four payer mix variables, one for each primary payer category (i.e., Medicare, Medicaid, commercial, and managed care). Lastly, hospitals were assigned a value from 1 to 4 depending on the quartile in which they resided for the distribution of each payer mix variable.

The AHA Annual Survey data include measures representing the percentage of each hospital's discharges that are Medicare and, separately, Medicaid. These variables were each broken into quartiles to align them with the NIS data we prepared. Because various organizational factors are associated with HIT adoption (Furukawa et al. 2008; Hikmet et al. 2008; Wang et al. 2005), each analysis we performed included control variables for the following hospital characteristics: bed size (measured as the natural log of the number of staff beds), system affiliation (yes or no), teaching status, geographic location (urban or rural), and tax status (for-profit or not-for-profit). These variables were derived from the AHA data. In addition, we controlled for case mix (defined as the average severity of patients treated at a given hospital) using the case mix index from the Medicare Cost Reports.

\section{Data Analyses}

All variables were examined for their distribution, suitability for analysis, and the existence of any potential data anomalies based on descriptive statistics. Next, we conducted chi-square analyses and independent-samples i-tests or analysis of variance (as appropriate) to identify any organizational differences between included and excluded hospitals, and we explored the univariate relationships between full EHR adoption and each variable measuring payer mix. Finally, using the NIS data, we examined the relationship between Medicare, Medicaid, commercial insurance, and managed care mix and EHR adoption while controlling for hospital characteristics and "other" types of discharges in a logistic regression model. We used the AHA data to examine a similar model with the obvious exclusion of the payer mix variables that do not appear in this data set (i.e., commercial insurance and managed care). In both the NIS and AHA models, we present EHR adoption as a binary variable (full EHR and partial EHR versus no EHR). In addition, we present the results of the NIS analysis specified as a multinomial regression that takes advantage of the categorical nature of the EHR variable. The results we present include adjusted odds ratios (ORs) and 95 percent confidence intervals for the logistic models, and beta coefficients for the multinomial regression model. Multivariate results are flagged for significance at the $?<0.05, \mathrm{p}<0.01$, and $?<0.001$ levels, respectively. In all regression models, we also controlled for the nested nature of hospitals clustered within states using Huber-White adjustments (Wooldridge 2003, 255) to our standard errors using the "clustered robust" command in STATA version 11.1. 


\section{RESULTS}

Organizational characteristics of included and excluded hospitals are displayed in Exhibit 1. Overall, NIS study hospitals $(n=392)$ had an average Medicare caseload of 47.9 percent, Medicaid caseload of 20.1 percent, commercial insurance caseload of 14.2 percent, and managed care caseload of 16.5 percent. Excluded NIS hospitals $(n=220)$ and AHA study hospitals $(n=$ 4,095 ) did not differ with respect to these payer mix variables (see Exhibit 1). Mean bed size for the NIS study sample was 205, significantly different than the excluded NIS hospitals (mean 143.2) and AHA study sample (mean 163.7; $\mathrm{p}$ 0.001). Overall, the NIS sample had a higher proportion of urban hospitals than excluded NIS hospitals and the AHA hospitals (62.4 percent vs. 41.6 vs. $49.9 ; \mathrm{p}<0.001$ ), but the three groups did not differ with respect to tax status, system affiliation, teaching status, or case mix index (see Exhibit 1).

In univariate analysis of the NIS data, Medicare discharges as a percentage of a hospital's payer mix was associated with EHR adoption (see Exhibit 2). Specifically, hospitals in the lowest quartile of Medicare discharges as a percentage of all discharges were more likely to have implemented a full EHR (19.1 percent vs. 3.1 percent; ? 0.001$)$ or a partial EHR $(55.9$ percent vs. 42.2 percent; $p<0.001$ ). No differences were observed in EHR adoption quartiles by Medicaid, commercial insurance, or managed care caseload. Increased hospital size, urban location, not-for-profit tax status, and teaching hospital status were positively associated with EHR adoption in univariate analyses (see Exhibit 2).

In multivariate analysis of the NIS sample and, separately, the AHA sample, controlling for payer mix, geographic location, tax status, bed size, system affiliation, teaching status, and case mix, virtually none of the payer mix variables were related to EHR adoption (see Exhibit 3). The only exception was that hospitals in the third quartile for managed care discharges (NIS sample only) were significantly less likely than those in the bottom quartile to report having an EHR $(\mathrm{OR}=0.18, ?=0.026)$. In the AHA model, several control variables, including geographic location, tax status, bed size, and system affiliation, were significantly associated with EHR adoption (see Exhibit 3).

Lastly, in the multinomial regression that examines the EHR adoption variable with three categories using the NIS sample, most of the payer mix variables were still not related to EHR adoption (see Exhibit 4). The only exception was the third quartile of managed care discharges, where hospitals in this category were again less likely to have adopted an EHR system (no EHR vs. full EHR beta $=2.289, \mathrm{p}=0.038$ ). 


\section{DISCUSSION}

While researchers have found that payer mix is associated with HIT adoption in hospitals, less attention has been paid to the association of payer mix and hospital EHR adoption. Given the federal government's provision of financial incentives to promote the adoption and meaningful use of EHR through the HITECH Act, we suggest that policymakers and hospital decision makers need a better understanding about the influence of payer mix on EHR adoption to fully realize the benefit of the legislation. In the absence of a previous analysis utilizing national data, we examine how the proportion of discharges paid by each payer is associated with EUR adoption by hospitals.

The findings in our current study suggest a weak relationship between payer mix and hospital EHR adoption. Even though we found that certain increases in Medicare caseloads were generally negatively associated with EHR adoption in univariate analysis, overall, these differences disappeared in our adjusted models, furthermore, most of the other payer mix variables were not associated with our outcome measure in any systematic way. These findings are in conflict with the existing studies that have found a relationship between individual HIT applications and payer mix (Furukawa et al. 2008). One possible explanation for this result may be that the adoption of an EHR system is differentially influenced by payer mix relative to the adoption of other types of HIT applications, full EHR adoption may be the final phase of HIT adoption in hospitals Thus, it is possible that payer mix influences the adoption of infrastructurerelated HIT applications such as pharmacy, laboratory, and radiology systems, but not complete EHR systems. If so, it is possible that the infrastructure-related HIT applications are less sensitive to resource availability. Perhaps the financial flexibility arising from serving patients from relatively generous payers may only apply to certain, not all, HIT decisions. This possibility would explain why we found a weak relationship between payer mix and hospital EHR adoption, whereas previous studies found a significant relationship between payer mix and the adoption of pharmacy and laboratory information systems (McCullough 2008) as well as other clinical, administrative, or strategic HIT applications (Furukawa et al. 2008; Menachemi et al. 2007). 
We expected that hospitals with higher proportions of public payer caseloads would be less likely to have an EHR system. We found no evidence to support this hypothesis in either the model using the NIS data or the model using the AHA payer mix data. Overall, this lack of evidence suggests the possibility that indirect incentives generated by payer generosity (as discussed earlier) may not be a strong factor influencing EHR adoption in hospitals. Under the HITECH program's direct incentives for EHR adoption, hospitals with comparably larger Medicare and Medicaid patient caseloads will be compensated proportionately higher for achieving meaningful use of EHRs. Thus, the HITECH Act has the potential, with some provisos, to assist in motivating hospitals that disproportionately serve Medicare and/or Medicaid patients to adopt an EHR and achieve meaningful use, thus fulfilling the intended objective of the legislation.

The first consideration for hospitals with high Medicare and/or Medicaid case mix is the level of HITECH program reward versus the total cost of ownership for an EHR. If the program's payback exceeds the financial cost of the EHR, then adopting a system is a rational choice. However, the program is designed to offset the EHR's purchase price and does not take into account the expenses associated with workflow redesign, temporary losses in productivity, and so forth (i.e., the total cost of ownership). Such analysis also may not consider potential quality gains associated with EHR use. Therefore, the decision to adopt an EHR is more complicated than merely to pursue the HITECH rewards. Considering discussions in a recent study that focused on ambulatory EHR adoption (Song et al. 2011 ), hospitals could benefit from considering the financial and nonfinancial benefits of EHRs when calculating the expected costbenefit ratio of pursing the incentive payments.

A second concern regarding hospitals with high Medicare and/or Medicaid caseloads relates to unintended consequences from the policymaker's perspective. The EHR incentive program may induce already undercapitalized hospitals to adopt a more leveraged position and face an increased risk of failure as they strive to meet the goals. Even if facilities choose not to invest in an EHR, the penalty phase of the meaningful use program may further cut into already faltering budgets. If these facilities are safety-net hospitals, the program may have the unintended consequence of hastening the failure of some of the very hospitals it is intended to assist and the concomitant impact on the most vulnerable populations in the United States. Of particular concern is the possibility that small, rural facilities will be adversely affected.

Our study also found that hospitals in the third highest category (out of four) as measured on the basis of managed care insurance caseloads were less likely than those with the lowest managed care caseloads to adopt EHR. While this finding is inconsistent with our hypotheses, it does not 
represent a systematic relationship between managed care caseloads and EHR adoption in hospitals. Thus, more research is needed to further understand this finding. On the other hand, several of our control variables were associated with EHR adoption in ways consistent with expectations based on previous HIT research. For example, in our study, urban hospitals (Burke et al. 2002; Furukawa et al. 2008), nonprofit hospitals (Menachemi et al. 2007), system-affiliated hospitals (Wang et al. 2005), and larger hospitals (Burke et al. 2002; Furukawa et al. 2008) were all more likely to have adopted EHRs.

The findings of this study offer practical implications for hospital decision makers and raise an important issue regarding national efforts imbedded in the HITECH Act. If payer generosity, or the indirect influence of payers, does not spur EHR adoption in hospitals, then the direct incentives in HITECH may represent the needed policy lever to influence EHR adoption. The important question becomes whether the direct incentives in the EHR adoption program will be motivation enough to overcome the resistance from some hospitals to begin the process of achieving meaningful use. Future research is needed to determine the full impact of the HITECH Act. Such research can utilize either the NIS or the AHA data to examine how Medicare and Medicaid caseloads are associated with EHR adoption after the HITECH Act has had more time to take hold. In the meantime, hospital decision makers should be aware that while the financial flexibility afforded by catering to more privately insured patients may enable the adoption of certain infrastructure HIT applications, such changes may not enable the adoption of EHRs.

The current study has several strengths. First, our topic is concerned with an important contemporary issue and makes use of a relatively large sample of hospitals potentially representative of US community hospitals. Furthermore, we make use of multiple data sources, which may help overcome common methods bias that negatively impacts the internal validity of studies using data extracted from a single source (lezzoni 2003). Despite these strengths, our analysis is limited in some aspects. First, given the cross-sectional observational nature of our analyses, we are unable to infer any causal relationship between payer mix and hospital EHR adoption. Therefore, our findings should be interpreted as associations only. Second, our sample size was inevitably decreased in the process of merging data sets and operationalizing our dependent and independent variables. We tried to overcome this limitation by running parallel analyses using a larger sample from the AHA Annual Survey, which contains less detailed payer information. The results of both analyses were similar. Lastly, our work is limited by the possibility of data entry and coding errors that can occur in secondary databases.

\section{References:}


Ali, ?. ?., H. S. Mekhjian, P. L. Kuehn, T. D. Bentley, R. Kumar, A. K. Ferketich, and S. P. Hoffmann. 2005. 'Specificity of Computerized Physician Order Entry Has a Significant Effect on the Efficiency of Workflow for Critically 111 Patients." Critical Care Medicine 33 (1): 110-14.

American Hospital Association (AHA). 2010. "Underpayment by Medicare and Medicaid Fact Sheet." Accessed January 7, 2012. www.aha.org/content/0010/ 10medunderpayment.pdf.

Blumenthal, D., and M. Tavenner. 2010. "The 'Meaningful Use' Regulation for Electronic Health Records." New England Journal of Medicine 363 (6): 501-4.

Boger, E. 2003. "Electronic Tracking Board Reduces ED Patient Length of Stay at Indiana Hospital." Journal of Emergency Nursing 29(1): 39-43.

Boxer, L. K., J. B. Dimick, R. M. Wainess, J. A. Cowan, P. K. Henke, J. C. Stanley, and G. R. Upchurch Jr. 2003. "Payer Status Is Related to Differences in Access and Outcomes of Abdominal Aortic Aneurysm Repair in the United States." Surgery 134 (2): 142-45.

Brailer, D. ). 2005. "Interoperability: The Key to the Future Health Care System." Health Affairs (Millwood) Web Exclusives, W5-19-W5-21.

Burke, D. E., B. B. Wang, T. T. Wan, and M. L. Diana. 2002. "Exploring Hospitals' Adoption of Information Technology." Journal of Medical Systems 26 (4): 349-55.

Centers for Medicare \& Medicaid Services (CMS). 2010. "CMS Finalizes Requirements for the Medicare Electronic Health Record (EHR) Incentive Program." U.S. Department of Health and Human Services. Published July 16. www.cms.gov/Apps/ Media/Press/Factsheet.Asp?Counter $=3792 \&$ Intnumperpage $=10 \&$ Checkdate $=\&$ Check key $=\&$ Srchtype $=1 \&$ Numdays $=3500 \&$ Srcho $\mathrm{pt}=0 \&$ Srchdata $=\&$ Keywordtype $=$ All $\&$ Chkn ewstype $=6 \&$ Intpage $=\&$ Showall $=\&$ Pyear $=\&$ Year $=\& D e s c=\&$ Cboorder $=$ Date.

Clement, J. P., and K. L. Grazier. 2001. "HMO Penetration: Has It Hurt Public Hospitals?" Journal of Health Care Finance 28 (1): 25-38.

Cleverley, W. O., and R. K. Harvey. 1992. "Competitive Strategy for Successful Hospital Management." Hospital \& Health Services Administration 37 (1): 53-69.

Dobson, A., J. Davanzo, and N. Sen. 2006. "The Cost-Shift Payment 'Hydraulic': Foundation, History, and Implications." Health Affairs (Millwood) 25 (1): 22-33.

Dor, A., and D. E. Farley. 1996. "Payment Source and the Cost of Hospital Care: Evidence from a Multiproduct Cost Function with Multiple Payers." Journal of Health Economics 15 (1): 1-21.

Friedman, B., N. Sood, K. Engstrom, and D. McKenzie. 2004. "New Evidence on Hospital Profitability by Payer Group and the Effects of Payer Generosity." International Journal of Health Care Finance and Economics 4 (3): 231-46. 
Furukawa, M. F., T. S. Raghu, T. J. Spaulding, and A. Vinze. 2008. "Adoption of Health Information Technology for Medication Safety in U.S. Hospitals, 2006." Health Affairs (Millwood) 27 (3): 865-75.

Garrido, T., B. Raymond, L. Jamieson, L. Liang, and A. Wiesenthal. 2004. "Making the Business Case for Hospital Information Systems - A Kaiser Permanente Investment Decision." Journal of Health Care Finance 31 (2): 16-25.

Hikmet, N., A. Bhattacherjee, N. Menachemi, V. O. Kayhan, and R. G. Brook. 2008. "The Role of Organizational Factors in the Adoption of Healthcare Information Technology in Florida Hospitals." Health Care Management Science 11 (1): 1-9.

Iezzoni, L. I. 2003. Risk Adjustment for Measuring Health Care Outcomes, 3rd ed. Chicago: Health Administration Press.

Jha, A. K., C. M. Desroches, E. G. Campbell, K. Donelan, S. R. Rao, T. G. Ferris, A. Shields, S. Rosenbaum, and D. Blumenthal. 2009. "Use of Electronic Health Records in U.S. Hospitals." New England Journal of Medicine 360 (16): 1628-38.

LaPar, D. ]., C. M. Bhamidipati, C. M. Mery, G. J. Stukenborg, D. R. Jones, B. D. Schirmer, I. L. Kron, and G. Ailawadi. 2010. "Primary Payer Status Affects Mortality for Major Surgical Operations." Annals of Surgery 252 (3): 544-50; discussion 550-51.

McCullough, J. S. 2008. "The Adoption of Hospital Information Systems." Health Economics 17 (5): 649-64.

McKay, N. L., and M. E. Deily. 2005. "Comparing High- and Low-Performing Hospitals Using Risk-Adjusted Excess Mortality and Cost Inefficiency." Health Care Management Review 30 [4): 347-60.

Mello, M. M., S. C. Stearns, and E. C. Norton. 2002. "Do Medicare HMOs Still Reduce Health Services Use After Controlling for Selection Bias?" Health Economics 11 (4): 323-40.

Menachemi, N., N. Hikmet, A. Bhattacherjee, A. Chukmaitov, and R. G. Brooks. 2007. "The Effect of Payer Mix on the Adoption of Information Technologies by Hospitals." Health Care Management Review 32 (2): 102-10.

Miller, R. H., and H. S. Luft. 2002. "HMO Plan Performance Update: An Analysis of the Literature, 1997-2001." Health Affairs (Millwood) 21 (4): 63-86.

Rosko, M. D. 2001. "Factors Associated with the Provision of Uncompensated Care in Pennsylvania Hospitals." Journal of Health and Human Services Administration 24 (3): 352-79. 
Russell, M. W., A. V. Joshi, P. J. Neumann, L. Boulanger, and J. Menzin. 2006. "Predictors of Hospital Length of Stay and Cost in Patients with Intracerebral Hemorrhage." Neurology 67 (7): 1279-81.

Song, P. H., A. S. McAlearney, J. Robbins, and I. S. McCullough. 2011. "Exploring the Business Case for Ambulatory Electronic Health Record System Adoption." Journal of Healthcare Management 56 (3): 169-80; discussion 181-82.

Wang, B. B., T. T. H. Wan, D. E. Burke, G. J. Bazzoli, and B. Y. J. Lin. 2005. "Factors Influencing Health Information System Adoption in American Hospitals." Health Care Management Review 30 (1): 44.

Wooldridge, J. M. 2003. Introductory Econometrics: A Modem Approach, 2nd ed. Cincinnati, OH: South-Western. 